\title{
Trending now
}

\section{Emerging methods in the tracking and prediction of infectious disease outbreaks}

Logan Van Nynatten, Ariel Gershon

Faculty Reviewer: Sameer Elsayed, MD, FRCPC (Division of Infectious Diseases)

\begin{abstract}
The threat of pandemic is looming in the public consciousness. Outbreaks of infectious diseases are tracked in order to respond to and prevent epidemics. Historically, both passive and active methods have been used. A promising development is to use social media, such as Twitter or Google, to track and predict outbreaks. Active monitoring consists of reporting and collection of health information, while passive monitoring involves studying data from clinicians to infer if an infectious outbreak has occurred. Social media provides a wealth of information to analyze outbreaks. By studying keywords searched on Google, researchers were able to predict outbreaks 1 to 2 weeks earlier than traditional methods used by the Centers for Disease Control.
\end{abstract}

\section{INTRODUCTION}

There are few events as frightening the as emergence of a previously undescribed infectious disease, or the re-emergence of a deadly pathogen. With the abundant and rapid access news media today, the fear and misinformation the public is subjected to compounds the chaos associated with outbreaks. Indeed, a study of 335 novel infectious diseases which have emerged globally between the years 1940 and 2004 showed that the rate of emerging infectious disease is increasing with time, even when taking into account increased surveillance of infectious diseases. ${ }^{1}$ Infectious disease pandemics (a global disease outbreak) and epidemics (a disease outbreak in a particular geographic location) are largely influenced by social, economic, and environmental factors, which supports the view that disease emergence is a non-random event. ${ }^{1}$ To contain public outbreaks, we have responded with many measures, including case and contact isolation (quarantine). ${ }^{2}$ However, to have a greater impact in preventing and monitoring emerging infectious diseases, we need to be able to track the spread of infection, and ideally, predict the populations where infection will appear nextreferred to as 'surveillance'. In the last decade, there has been a push to integrate our technological advances with our knowledge of infectious diseases in order to predict when pandemics may occur. The culmination of these efforts has resulted in public health authorities being able to link certain population activities with outbreaks, a development that has more recently exploited our use of social media to help track pandemics.

\section{METHODS OF INFECTION SURVEILLANCE}

Historically, active or passive methodologies have been used for infectious disease surveillance at a global level. Active surveillance involves monitoring disease by proactively collecting information, usually for those diseases where infection remains subclinical or asymptomatic. For example, testing for pulmonary tuberculosis infection via respiratory cultures is active surveillance for infection. ${ }^{3} \mathrm{On}$ the other hand, passive surveillance involves routinely collecting health data to monitor infection and allows one to make inferences and predictions about the activity of certain infectious diseases, and remains a common method of disease surveillance. ${ }^{4}$ Often passive surveillance is the method of choice for infectious disease monitoring as it is significantly less expensive compared to active surveillance programs.

\section{WITH 271 MILLION ACTIVE USERS AND AN AVERAGE OF 500 MILLION TWEETS DAILY, TWITTER IS AN IMMENSE SOURCE OF INFORMATION THAT MAY BE APPLIED TO DISEASE SURVEILLANCE.}

Passive surveillance involves the reporting of cases of patients with infections by health care practitioners to administrative bodies. For example, the Ontario Ministry of Health 2013 report demonstrates the passive surveillance methods that are employed in Ontario to monitor influenza. ${ }^{5}$ Some of these methods include: collecting community reports of influenza cases, critical care reports, emergency department reports, and hospitalization records related to influenza. Unfortunately, using healthcare providers to collect information means results are dependent on those infected individuals who actually seek treatment, which for most infections are older adults or young children. ${ }^{6,7}$ Several different modalities of disease surveillance are required to ensure a complete picture of the state of infection in a population is captured.

In addition to traditional methods of surveillance, other methods of tracking infection have been explored. Pharmacy sales of over-the-counter medications and prescription medications may be used to track both gastrointestinal and respiratory infections. ${ }^{8}$ In fact, data from pharmacy sales can be used to on average predict influenza outbreaks in communities 2.8 days before data generated from physician reports. ${ }^{9}$ Furthermore, the Ontario telehealth program has been used to monitor the spread of such infections like influenza, and the data collected has been used to predict influenza activity 1 to 2 weeks earlier as compared to data obtained from healthcare reports. ${ }^{10,11}$ Data collected from student or employee absenteeism have also been used to predict when outbreaks will occur several weeks earlier as compared to traditional surveillance and 
laboratory programs. ${ }^{12,13}$

The goal of infection surveillance methods is to identify as many infected individuals as possible-as early on in infection as possible-and being able to accurately use this information to track and predict how infection will spread throughout the population.

\section{THE EMERGENCE OF SOCIAL MEDIA IN SURVEILLANCE OF INFECTION}

As both the global population and ease of intercontinental travel increase, the likelihood of a life-threatening pathogen sweeping across the globe grows within the realm of possibility and public fears. To date, a number of infections have spread across the globe, such as the human immunodeficiency virus (HIV) epidemic in the 1980s, the severe acute respiratory virus (SARS) outbreak of 2002, and the recent Ebola virus epidemics in 2014 and 2015. These are only some of many pathogens that have ravaged the human population, with the next 'superbug' looming in the not-so-distant future. Hence, the methods that we use to track infectious disease must continue to evolve so that we will be able to deal with the threat of the next pandemic. Over the last decade, the promise of social media-with its reach far beyond any traditional surveillance method-has arisen as a potential tool that we may take advantage of to track and predict infectious disease outbreaks amongst the global community. ${ }^{8,14}$ In fact, it has been shown that there is significant attention to infectious diseases outbreaks on social media, as has occurred with Middle Eastern respiratory syndrome coronavirus and avian influenza. ${ }^{15}$

Twitter is one social media outlet that has been explored for this purpose. With 271 million active users and an average of 500 million tweets daily, Twitter is an immense source of information that may be applied to disease surveillance. The general idea is based on the premise that screening 'tweets' posted by infected individuals for certain keywords associated with their infection can be used to determine where people are infected geographically, at what time, and where the infection may be likely to have a high public health burden. ${ }^{16,17}$ For example, tweets containing the words such as "flu", "fever", "sore throat", and "cough" have been shown to be associated with influenza activity. ${ }^{18}$ Indeed, the H1N1 epidemic of 2009 was tracked in such a way, and the movement of the virus was monitored in real time across the United States using Twitter metadata. This had greater predictive probability when compared to traditional reports from the Centers for Disease Control (CDC). ${ }^{19,20}$ Remarkably, infection tracking in this way could predict outbreaks one week earlier than the CDC. ${ }^{20}$

Similarly, Google keyword searches can be analyzed to predict infection outbreaks. ${ }^{21}$ There is a relationship between those people searching for influenza related information on Google, and those individuals who have influenza symptoms and infection. ${ }^{21}$ Collecting such data this way has demonstrated to be a robust, timely, and sensitive method of infection surveillance, with Google Flu Trends accurately predicting outbreaks on average 1 to 2 weeks earlier than the reports published by the CDC. Similar results have been observed when tracking cases of West Nile Virus infection. ${ }^{21}$ Further- more, correlations have been established between internet AdSense advertisements or searches regarding flu symptoms, with cases of influenza. 22,23 Moreover, screening of blog posts released on the internet for infection related content can assist in tracking disease outbreaks. ${ }^{24} \mathrm{~A}$ strong co-occurrence has been reported between the frequency of such posts and keywords, with influenza cases. ${ }^{24}$

\section{FUTURE DIRECTIONS}

With the wealth of 'big data' available using social media outlets to track and predict infectious disease outbreaks, there is a need to further refine computational analysis to effectively sort and collect this information. ${ }^{25}$ Further studies are needed to strengthen the evidence for the reliability and quality of data obtained via social media surveillance. Additionally, ethical considerations of collecting data through the use of social media must also be addressed to ensure confidentiality and privacy. ${ }^{25}$ It is also possible to apply these methods to coordinate the public health response to outbreaks. Social media may be used to provide warnings to the public, coordinate relief efforts, and promote social cohesiveness in times of infectious diseases outbreaks. ${ }^{26,27}$ For example, during the 2009 H1N1 pandemic, the Alexandria, Virginia health department tweeted where vaccines were available, effectively managing vaccine distribution. ${ }^{28}$ Social media is useful in not only predicting outbreaks, but also managing the public health response to them.

\section{CONCLUSIONS}

It is clear that additional methods of infectious disease surveillance will assist in both the prediction of and response to outbreaks. In order to better monitor infections, several modalities of surveillance will be needed to generate a complete understanding of the epidemiology of infection across populations and demographics. The abundance of information available on social media represents an exciting avenue to predict and track infectious diseases outbreaks. We must use interdisciplinary and novel methods to prepare for an overwhelming global pandemic-a situation we are relatively ill-prepared for. Social media is a novel defense mechanism in this ever-escalating arms race between pathogen and hosts.

\section{REFERENCES}

1. Jones KE, Patel NG, Levy MA, et al. Global trends in emerging infectious diseases. Nature. 2008 Feb 21;451(7181):990-3.

2. Fraser C, Riley S, Anderson RM, Ferguson NM. Factors that make an infectious disease outbreak controllable. Proc Natl Acad Sci U S A. 2004 Apr 20;101(16):6146-51.

3. Menzies D, Fanning A, Yuan L, FitzGerald JM. Hospital ventilation and risk for tuberculous infection in Canadian health care workers. Ann Intern Med. 2000 Nov 21;133(10):779-89.

4. Thacker SB, Choi K, Brachman PS. The surveillance of infectious diseases. JAMA. 1983 Mar 4;249(9):1181-5.

5. Ontario Health Plan for an Influenza Pandemic [Internet]. Ministry of Health and Long-Term Care; March 2013 [Accessed February 18 2016]. Available from: http://www.health.gov.on.ca/en/pro/programs/emb/ pan_flu/docs/ch_03.pdf.

6. Baer A, Rodriguez CV, Duchin JS. An automated system for public health surveillance of school absenteeism. J Public Health Manag Pract. 2011 Jan-Feb;17(1):59-64. 
7. Moore K. Real-time syndrome surveillance in Ontario, Canada: the potential use of emergency departments and Telehealth. Eur J Emerg Med. 2004 Feb;11(1):3-11.

8. Patwardhan A, Bilkovski R. Comparison: flu prescription sales data from a retail pharmacy in the US with Google Flu trends and US ILINet (CDC) data as flu activity indicator. PLoS One. 2012;7(8):e43611.

9. Magruder SF. Evaluation of over-the-counter pharmaceutical sales as a possible early warning indicator of human disease. J Hopkins Apl Tech D. 2003;24:349-53.

10. Cooper D, Smith GE, Edmunds WJ, et al. The contribution of respiratory pathogens to the seasonality of NHS Direct calls. J Infect. 2007 Sep;55(3):240-8.

11. Cooper DL, Smith GE, Regan M, et al. Tracking the spatial diffusion of influenza and norovirus using telehealth data: a spatiotemporal analysis of syndromic data. BMC Med. 2008 Jun 26;6:16.

12. Van den Wijngaard C, van Asten L, van Pelt W, et al. Validation of syndromic surveillance for respiratory pathogen activity. Emerg Infect Dis. 2008 Jun;14(6):917-25.

13. Van den Wijngaard C, van Pelt W, Nagelkerke N, et al. Evaluation of syndromic surveillance in the Netherlands: its added value and recommendations for implementation. Euro Surveill. 2011 Mar 3;16(9). pii: 19806.

14. Aslam AA, Tsou MH, Spitzberg BH, et al. The reliability of tweets as a supplementary method of seasonal influenza surveillance. J Med Internet Res. 2014 Nov 14;16(11):e250.

15. Fung IC, Fu KW, Ying Y, et al. Chinese social media reaction to the MERS-CoV and avian influenza A (H7N9) outbreaks. Infect Dis Poverty. 2013 Dec 20;2(1):31.

16. Schmidt CW. Using social media to predict and track disease outbreaks. Environ Health Perspect. 2012 Jan;120(1):A30-3.

17. St Louis C, Zorlu G. Can Twitter predict disease outbreaks? BMJ. 2012 May 17;344:e2353.

18. Szomszor M, Kostkova P, De Quincey E. \#Swineflu: Twitter predicts swine flu outbreak in 2009. In: Szomszor M, Kostkova P, eds. Electronic Healthcare. Heidelberg, Germany: Springer-Verlag GmbH; 2010. p. 18-26.

19. Signorini A, Segre AM, Polgreen PM. The use of Twitter to track levels of disease activity and public concern in the US during the influenza A H1N1 pandemic. PLoS One. 2011 May 4;6(5):e19467.

20. Paul MJ, Dredze M, Broniatowski D. Twitter improves influenza forecasting. PLoS Curr. 2014 Oct 28;6.

21. Carneiro HA, Mylonakis E. Google trends: a web-based tool for real-time surveillance of disease outbreaks. Clin Infect Dis. 2009 Nov 15;49(10):1557-64.

22. Eysenbach G. Infodemiology: tracking flu-related searches on the web for syndromic surveillance. AMIA Annu Symp Proc. 2006:244-8.

23. Eysenbach G. Infodemiology and infoveillance: framework for an emerging set of public health informatics methods to analyze search, communication and publication behavior on the Internet. J Med Internet Res. 2009 Mar 27;11(1):ell.

24. Corley CD, Cook DJ, Mikler AR, Singh KP. Text and structural data mining of influenza mentions in web and social media. Int J Environ Res Public Health. 2010 Feb;7(2):596-615.

25. Moorhead SA, Hazlett DE, Harrison L, et al. A new dimension of health care: systematic review of the uses, benefits, and limitations of social media for health communication. J Med Internet Res. $2013 \mathrm{Apr}$ 23;15(4):e85.

26. Houston JB, Hawthorne J, Perreault MF, et al. Social media and disasters: a functional framework for social media use in disaster planning, response, and research. Disasters. 2015 Jan;39(1):1-22.

27. Imran M, Elbassuoni SM, Castillo C, et al. Extracting information nuggets from disaster-related messages in social media. Proc 10th Int ISCRAM Conf. 2013 May:1-10.

28. Merchant RM, Elmer S, Lurie N. Integrating social media into emergency-preparedness efforts. N Engl J Med. 2011 Jul 28;365(4):289-91. 